\title{
Diagnóstico higiénico-sanitario e identificación de factores de riesgo en restaurantes
}

\section{Hygienic-sanitary diagnosis and risk factors identification in restaurants}

CAMPOS-BRAVO, Carlos Alberto†*, JIMÉNEZ-ORTEGA, Luis Alfonso, GONZÁLEZ-AGUILAR, Delia Guillermina y BARBARO, Bárbara

Universidad de Guadalajara. Centro Universitario de Ciencias Biológicas y Agropecuarias. Departamento de Salud Pública. Camino Ramón Padilla Sánchez, 2100, 45200 Nextipac, Zapopan, Jalisco, México.

ID $1^{\mathrm{er}}$ Autor: Carlos Alberto, Campos-Bravo / ORC ID: 0000-0002-3981-5608, Open ID: 109025180370696050835

ID $1^{\text {er }}$ Coautor: Luis Alfonso, Jiménez-Ortega / ORC ID: 0000-0002-9861-8911

ID $2^{\text {do }}$ Coautor: Delia Guillermina, González-Aguilar / ORC ID: 0000-0003-1413-2330

ID $3^{\text {er }}$ Coautor: Bárbara, Barbaro / ORC ID: 0000-0002-7495-1592

DOI: $10.35429 / J O H S .2019 .21 .6 .14 .23$

Recibido Octubre 10, 2019; Aceptado Diciembre 29, 2019

\section{Resumen}

El objetivo fue realizar un diagnóstico higiénico-sanitario y la identificación de factores de riesgo en 81 establecimientos fijos de restauración de alimentos ubicados en el área metropolitana de Guadalajara, Jalisco, México. Los establecimientos se agruparon según el Sistema de Clasificación de la Industria de América del Norte (SCIAN). Se aplicó el acta de verificación sanitaria, basada en la NOM-251-SSA1-2009. El mínimo para considerar aprobada la evaluación fue del $85 \%$. 26 lugares aprobaron la evaluación, los tres mejor calificados, pertenecen a franquicias, 55 establecimientos fueron reprobados. Estadísticamente representa lo mismo que el servicio corresponda a cualquiera de las categorías SCIAN abordadas en el estudio. Los factores de riesgo detectados en orden de importancia están asociados a los siguientes programas: control de calidad del agua de uso general; capacitación/ entrenamiento del personal; Transporte; control de plagas; control de operaciones; mantenimiento y limpieza; Instalaciones y áreas; control de calidad del agua en contacto con alimentos. Se evidenció la ausencia escrita y operativa de programas prerequisitos, por lo que las condiciones y medidas aplicadas no son suficientes para asegurar la calidad y la inocuidad de los alimentos y por tanto representan un riesgo potencial de enfermedad para los comensales en los restaurantes locales.

Servicios de restauración alimenticia, Factores de riesgo, Programas pre-requisitos

\begin{abstract}
The purpose was to make a hygienic-sanitary diagnosis and risk factors identification in 81 fixed establishments of food restoration located in the metropolitan area of Guadalajara, Jalisco, Mexico. The establishments were grouped according to the North American Industry Classification System (NAICS). The sanitary verification act, based on NOM-251-SSA1-2009, was applied. The minimum was $85 \%$ in order to consider the evaluation was approved. 26 places approved the evaluation, the three best qualified, belong to franchises, 55 establishments were failed. Statistically it represents the same as the service corresponds to any of the NAICS categories addressed in the study. The risk factors detected in order of importance are associated with the following programs: quality control in water for general use; staff training; transport; pest control; operations control; maintenance and cleaning; Facilities and areas; quality control of the water in contact with foods. The written and operational absence of prerequisite programs was evidenced, so the conditions and measures applied are not sufficient to ensure the quality and safety of the food, and therefore represent a potential disease risk for diners in local restaurants.
\end{abstract}

Food restoration services, Risk factors, Prerequisite programs

Citación: CAMPOS-BRAVO, Carlos Alberto, JIMÉNEZ-ORTEGA, Luis Alfonso, GONZÁLEZ-AGUILAR, Delia Guillermina y BARBARO, Bárbara. Diagnóstico higiénico-sanitario e identificación de factores de riesgo en restaurantes. Revista de Ciencias de la Salud. 2019. 6-21: 14-23.

\footnotetext{
*Correspondencia al Autor (Correo Electrónico: carlos.cbravo@academicos.udg.mx)

$\dagger$ Investigador contribuyendo como primer Autor
} 


\section{Introducción}

En México, la unidad responsable de llevar a cabo la tarea de verificar el cumplimiento de las normas de higiene en los restaurantes es la Secretaría de Salud (SS), a través de la Comisión Federal para la Protección contra Riesgos Sanitarios (COFEPRIS), con base en las regulaciones nacionales que establecen los estándares de inocuidad (Pérez, Lizárraga y Medina, 2018), pero con las limitantes de un campo de acción muy amplio y pocos verificadores sanitarios, además de que por lo que se conoce, los datos recabados no son analizados para generar estrategias de intervención. La industria restaurantera es la segunda con más empleos en México, donde predominan los micro establecimientos (97.8\%), que representan el $77.7 \%$ del personal empleado (Instituto Nacional de Estadística y Geografía [INEGI], 2014), lo cual refleja el impacto potencial que estos servicios de alimentos pueden tener en la salud del consumidor final. La importancia cultural y económica de la industria restaurantera en México es innegable (Vera y Trujillo, 2017), en este contexto, el conocimiento de los factores de riesgo asociados con los servicios de alimentos en la zona metropolitana de Guadalajara, Jalisco, México, aunque se conoce empíricamente, carece de fundamentos en investigaciones de campo, por lo que el objetivo de este estudio fue hacer un diagnóstico higiénico-sanitario e identificar la aparición de factores de riesgo y prácticas inseguras asociadas con enfermedades transmitidas por los alimentos (ETA) en los servicios de alimentos para posteriormente contribuir al diseño de programas de intervención que minimicen su incidencia. La hipótesis planteada es: si prevalecen las deficientes condiciones higiénico-sanitarias en los servicios de alimentos solo el $25 \%$ de los establecimientos aprueban la verificación sanitaria.

\section{Evaluación de los servicios de alimentos}

Los alimentos poco saludables generan un círculo vicioso de enfermedades y mala nutrición, además de tener repercusiones económicas, sociales, políticas y turísticas, lo que dificulta el desarrollo de las personas (Kopper, Calderón, Shneider, Domínguez y Gutiérrez, 2009; World Health Organization [WHO], 2016; WHO, 2019).
El consumo de alimentos fuera del hogar ha sido uno de los cambios importantes en el estilo de vida en las últimas décadas (Lachat et al., 2012; Powell, Nguyen, y Han, 2012). Junto con estos cambios, los comportamientos del personal que afectan la inocuidad alimentaria y repercuten en la aparición de enfermedades en el consumidor, asociados con estos establecimientos, deben ser vigilados más de cerca (Food and Drug Administration [FDA], 2018).

En todo el mundo, los servicios de alimentos han sido reportados como lugares de origen ETA, los más frecuentemente mencionados son: restaurantes, bares, escuelas, guarderías, hogares de ancianos y comedores colectivos, que involucran alimentos de origen animal y vegetal, asociados con indeseables condiciones en el proceso de producción y que implican contaminación cruzada (Angelo, Nisler, Hall, Brown y Gould., 2017); Angulo, Jones y Angulo, 2006; Leinwand, Glanz, Keenan y Branas, 2017; Zavala, 2015).

El Centro para el Control y la Prevención de Enfermedades (CDC, por sus siglas en inglés) informa que entre 1998 y 2004 en los EE. UU, el $52 \%$ de los brotes de ETA se originó en restaurantes (con un aumento en restaurantes étnicos), $18 \%$ en hogares y $4 \%$ en escuelas (Angulo et al., 2006).

En Chile, el $29.3 \%$ de los brotes se originaron en el hogar y el $12.8 \%$ en restaurantes; El $11.3 \%$ se asoció con inocuidad ausente en el proceso de producción (Olea, Díaz, Fuentes, Vaquero y García, 2012). En el estudio realizado por DiPietro et al. (2004), el 23\% de los restaurantes están asociados con la aparición de enfermedades, el $8 \%$ en restaurantes de hoteles y el $5 \%$ en comedores escolares, con el denominador común de mala higiene.

Los CDC mencionan cinco categorías de alimentos asociados con la enfermedad: carne de aves de corral $33 \%$, verduras $25 \%$, huevos-leche $23 \%$, acuáticos $12 \%$ y frutas-nueces $7 \%$, en las que destacan: carne de res, leche, frijoles y aves de corral (Nsoesie, Kluberg y Brownstein, 2014), que se encuentran con frecuencia en los servicios de restauración de alimentos. Esto coincide con lo expresado por otros autores (Flórez, Rincón, Garzón, Vargas y Enríquez, 2008; Kopper et al., 2009; Ole et al., 2012). 
Para la Administración de Alimentos y Medicamentos (FDA, por sus siglas en inglés), los factores de riesgo asociados con las ETA en los servicios de alimentos son: 1. mala higiene del personal; 2. tiempo y temperatura de almacenamiento inadecuados; 3. equipo contaminado; 4. cocción inadecuada; 5. alimentos de fuentes inseguras (FDA, 2018).

La investigación de Flórez et al. (2008), reporta $8.3 \%$ de los restaurantes con ubicación inadecuada, $37.7 \%$ sin planes de saneamiento y 91.3\% con prácticas de almacenamiento incorrectas. En México, Zavala (2015), al auditar servicios de alimentos, revela que la mayoría de los aspectos tenían un cumplimiento parcial, aquellos con el mejor cumplimiento fueron: Infraestructura; Equipos y utensilios. Con más fallas en el cumplimiento fueron: Capacitación; Control de plagas; Limpieza y desinfección.

Las verificaciones llevadas a cabo por la autoridad tienen el propósito de proteger a la población contra los riesgos para la salud y tener un impacto en la disminución de las ETA Pérez et al., 2018; Reske, Jenkins, Fernandez, VanAmber, y Hedberg, 2007). Es bien sabido que en los países en desarrollo las medidas destinadas a controlar la seguridad alimentaria se implementan con mayores o menores dificultades (Kopper et al., 2009).

Entre esas medidas, la implementación de programas prerrequisitos (PPR), conduce a la producción de alimentos inocuos debido a la reducción de las cargas bacterianas (Garayoa, Yánez, Díez, Bes, y Vitas, 2016; Rodrigues, Silva, y Aleixo, 2012).

Las medidas de control a través de los PPR y HACCP se han informado como una estrategia positiva para la inocuidad alimentaria, pero su implementación deficiente es un precursor de la aparición de brotes de ETA (Cormier, Mallet, Chiasson, Magnússon y Valdimarsson, 2007; Luning et al., 2009).

\section{Metodología a desarrollar}

Se realizó un estudio observacional en servicios de alimentos. Las evaluaciones se aplicaron a 81 servicios fijos de preparación, venta y consumo de alimentos ubicados en el área metropolitana de Guadalajara, Jalisco, México, que emitieron su autorización para llevarlas a cabo.
Los establecimientos se agruparon según las categorías de servicios de preparación de alimentos y bebidas, establecidos en el Sistema de Clasificación de la Industria de América del Norte (SCIAN) (INEGI, 2018).

Los datos se recopilaron en una sola visita por establecimiento, a través del acta de verificación de higiénico-sanitaria, emitida por COFEPRIS, basada en la NOM-251-SSA1-2009 (SS, 2010); Se tomaron en cuenta las secciones I (84 ítems), "Disposiciones generales de establecimientos" y III (46 ítems), "Disposiciones aplicables a los establecimientos de servicios de alimentos y bebidas". Se abordaron los siguientes aspectos: Instalaciones y áreas, equipo y utensilios, servicios, almacenamiento, control de operaciones, materias primas, empaque, agua en contacto con alimentos, mantenimiento y limpieza, control de plagas, manejo de residuos, salud e higiene del personal, transporte, así como registros y documentación, todos ellos relacionados con la implementación de programas de prerrequisitos.

La calificación se otorgó en base a los siguientes criterios: (2), cumple totalmente; (1), cumple parcialmente; (0), no cumple; (--), no aplica. La guía emitida por COFEPRIS se utilizó para evaluar el cumplimiento del acta de verificación, donde los ítems se clasifican como: $\mathrm{R}$, Punto requerido de buenas prácticas (aspectos básicos de inocuidad); C, Punto crítico de Inocuidad (aspectos intermedios de inocuidad); $\mathrm{C}+$, Punto crítico de Inocuidad Indispensable (aspectos de inocuidad indispensables). La aprobación mínima fue del $85 \%$ (COFEPRIS, 2017).

Se realizaron pruebas ANOVA y HSD Tuckey (Statgraphics®, 2013), agrupadas por: categoría SCIAN, sección del acta y categoría de calificación ( $\mathrm{R}, \mathrm{C}$ y $\mathrm{C}+$ ). La significancia estadística fue aceptada con un nivel de confianza del $95 \%$.

\section{Resultados}

El valor tangible de la inocuidad alimentaria es a través de la aplicación de los programas prerrequisitos, sin embargo, el conocimiento insuficiente o inadecuado sobre ellos se refleja en los resultados obtenidos, lo que conduce a fallas en el manejo de los alimentos como la contaminación cruzada. 


\section{Clasificación SCIAN y categoría de establecimientos}

Estadísticamente, las diferentes categorías SCIAN ( $\mathrm{p} \geq 0.05)$ son iguales, tanto al analizar por sección del acta como por categoría de calificación (R, C, C +). 26 (32.1\%) de los 81 establecimientos aprobaron la evaluación (tabla 1). En la clase de actividad 722511 , los puntajes de los 13 servicios de alimentos aprobados variaron de 85.55 a 94.35. En SCIAN 722512, los dos aprobados obtuvieron 89.66 y 90.68. Un restaurante que vende "tortas" fue calificado con 90.91 (SCIAN 722514).

En SCIAN 722515, los tres lugares aprobados calificaron con 90.43, 90.83 y 91.6. Los restaurantes con venta de hamburguesas SCIAN 722517 (aprobados tres de tres), presentaron las calificaciones más altas de todo el estudio (94.78, 95.3 y 98.74), pertenecen a franquicias que tienen programas de prerrequisitos bien establecidos y que a menudo son preferidos por los comensales, esto coincide con lo señalado por Harris, DiPietro, Murphy y Rivera (2014). Las cuatro pizzerías aprobadas fueron evaluadas entre 85.47 y 93.5, una de las cuales también es franquicia.

En el presente estudio, el 55 (67.9\%) de los lugares auditados fueron reprobados (Cubren todas las clases de actividad) (tabla 1), los límites de calificación fueron: 31.40 (SCIAN 722511) y 84.87 (SCIAN 722512). Cabe señalar que en la actividad SCIAN 722513 (antojitos), los nueve establecimientos evaluados no aprobaron (calificados entre 43.1 y 81.25), así como seis de siete restaurantes en SCIAN 722514 (tacos/tortas), calificados entre 46.98 y 77.23 . Leinwand et al. (2017), mencionan que las pequeñas empresas representan un mayor riesgo de transmitir enfermedades debido a su dificultad en la implementación de programas de aseguramiento de la inocuidad.

Estos establecimientos son lugares que los comensales suelen visitar, lo que está asociado con la cultura de los mexicanos, por lo que el riesgo potencial asociado con las deficiencias de estos y otros lugares reprobados es alto.
Harris, Murphy, DiPietro, y Rivera, (2015) señalan que los restaurantes que ofrecen alimentos tradicionales son aquellos que representan los principales factores de riesgo para transmitir enfermedades debido a la gran cantidad de desviaciones críticas que presentan, lo cual coincide con el presente estudio y con lo señalado por Muguruza (2019), la cultura de inocuidad es deficiente en los negocios que participaron en una feria gastronómica.

Si la verificación hubiera sido realizada por la Secretaría de Salud, deberían ser suspendidos por incumplimiento, sin embargo, este porcentaje difiere con lo informado por COFEPRIS, que en 2015 y el primer semestre de 2016, llevó a cabo 98,402 inspecciones sanitarias en los servicios de alimentos, de las cuales solo $946(0,96 \%)$ fueron suspendidas por incumplimiento (Pérez et al., 2018).

El abordaje de la gestión a través de ISO, permite una integración total de los sistemas individuales, con la reducción de la información necesaria, cumpliendo con la normatividad y permitiendo a las empresas aspirar a adquirir la certificación en Buenas Prácticas de Manufactura (Rojas, 2019).

A este respecto, la gran mayoría de los establecimientos incluidos en el estudio no tienen sistemas de gestión de inocuidad alimentaria (FSMS), a este respecto, la FDA (2018), señala que los establecimientos con FSMS ineficiente tienen prácticas significativamente más inapropiadas, lo que se refleja en el estudio (tablas 1, 2).

\section{Evaluación a través de las secciones del acta de verificación sanitaria}

La aplicación de una lista de verificación es auxiliar importante para determinar conformidad del cumplimiento de la normatividad, Pilco (2019), determinó el $61 \%$ de conformidad, en el Salad Bar de un supermercado y ubica al establecimiento dentro de la calificación "en proceso", requiriendo para ello acciones de gestión. 
En el presente estudio, la evaluación de las secciones del acta con respecto a $\mathrm{R}, \mathrm{C}$ y $\mathrm{C}+$, no presenta diferencias estadísticas significativas $(p \geq 0.05)$, lo que si ocurre al analizar las secciones del registro de acuerdo con las categorías SCIAN $(p \leq 0.05)$. El mayor porcentaje de cumplimiento fue presentado por las siguientes secciones del registro: equipo y utensilios $(87.59 \%)$; materias primas $(84.33 \%) \mathrm{y}$ control de plagas $(83.31 \%)$.

La ausencia de registros, asociada con la falta de programas prerrequisitos (debido a la falta de conocimiento de los propietarios o gerentes de los restaurantes), es evidente en los que se considera son los principales factores de riesgo asociados a las secciones del acta de verificación, en orden de importancia:

1. Registros de control de agua (10.64\% de cumplimiento). La deficiencia en el control documental sobre la vigilancia de cloro residual libre y organismos coliformes fecales y totales en el agua potable destinada a la limpieza y desinfección de instalaciones y, a menudo, de frutas y verduras, representa un riesgo inminente de contaminación cruzada debido a la posible presencia de organismos coliformes fecales. Incluso si esporádicamente se llevan a cabo acciones de cloración del agua (sin lavado previo de los tanques de agua).

2. Registros de entrenamiento (47.85\%). Falta de evidencia con respecto a la capacitación del personal (que a menudo no ha ido más allá de la educación secundaria), sobre los programas prerrequisitos. Esto implica no solo la ausencia del registro, sino también la falta del programa específico y la capacitación por personas sin competencia técnica, lo que redunda en una pobre capacitación del personal.

3. Transporte $(49.15 \%)$. El transporte de materias primas no se realiza en vehículos específicos, que no están hechos de material resistente, liso, impermeable o fácil de limpiar. Los productos que requieren refrigeración o congelación no se transportan a las temperaturas de almacenamiento recomendadas.
4. Registros de control de plagas (58.13\%). Muy pocos establecimientos tienen un programa específico para el control de plagas o presentan la licencia sanitaria de la empresa que brinda el servicio, por lo tanto, no hay confianza de que sean personal autorizado por la Secretaría de Salud, ni la certeza de que se usan pesticidas autorizados, con el consiguiente riesgo para los comensales. Es común que se empleen plaguicidas de libre venta en el comercio, lo cual está asociado a que en la sección control de plagas manifestaron un $83.31 \%$ de cumplimiento.

5. Control de operaciones (60.24\%). El control de las operaciones asociado con las temperaturas de recepción en frío (refrigerados y congelados), y las temperaturas de cocción o recalentamiento, generalmente se llevan a cabo de manera inconsciente y subjetiva, es decir, sin el uso de un termómetro, lo que aumenta la probabilidad de ocurrencia de riesgos asociados a la proliferación bacteriana, junto con la ausencia de otros controles organolépticos. Aunque, por otro lado, la experiencia de los empleados proporciona una falsa seguridad ya que no son realmente conscientes de la importancia de estandarizar este aspecto.

En ocasiones hay cruce de procesos; Uso indistinto de utensilios para probar los alimentos o bebidas; Los productos fuera de especificación no están marcados o separados en condiciones apropiadas.

6. Mantenimiento y limpieza (69.30\%). Procedimientos inadecuados de limpieza y desinfección de manos, platos, vasos, cubiertos, equipos y utensilios; Uso de trapos en condiciones de higiene inadecuadas, para secar platos y cubiertos.

Muguruza (2019), describe a la falta de aplicación de las Buenas Prácticas de Manufactura, sobre todo a limpieza y desinfección como el factor de riesgo que más se asocia a la presencia de S. aureus, E, coli, y coliformes totales en los alimentos. 
La aplicación efectiva de procedimientos de limpieza y desinfección inciden en la inocuidad de los alimentos al no encontrar después de 8 meses de su implementación la presencia de Escherichia coli, afirma Pilco (2019).

7. Instalaciones y áreas (69.41\%). Diseño inadecuado de instalaciones que favorecen la contaminación de materias primas y / o productos (p.ej. empalmes pared-pared y pared-piso que forman un ángulo, puertas y ventanas sin protección); dificulta los procedimientos de limpieza y desinfección; favorece la entrada de lluvia y fauna nociva, lo que crea un círculo vicioso que conduce a la persistencia de las plagas.

8. Agua en contacto con los alimentos (69.94\%). Contrasta con el aspecto de menor cumplimiento que fue registro de control del agua, por lo que en realidad es importante considerar que este tipo de agua no es tan confiable cuando se usa de la red municipal. Sin embargo, cuando es agua embotellada si lo es.

Los hallazgos del presente estudio, en general coinciden con lo indicado por Zavala (2015), quien detectó que los factores de riesgo más relevantes son infraestructura, agua para uso y consumo humano, contaminación directa y cruzada, control de temperatura del buffet y fauna nociva. Además coincide con los factores de riesgo mencionados por la FDA (2018), y con los hallazgos de Djekic et al. (2014) y Rodrigues et al. (2012).

La autoridad en México, necesariamente requiere un curso de capacitación, sin embargo, es evidente que no ha tenido el efecto deseado ya que la mayoría de los restaurantes cumplen parcialmente con las secciones del informe de verificación y tienen serias deficiencias en relación con el registros.

\section{Cumplimiento del acta (R, C, C +)}

Los establecimientos auditados se centran más en cumplir con los aspectos intermedios relacionados con la inocuidad (C), que representa la sección con el mayor número de elementos $(44.14 \%)$, incluye aspectos que pueden clasificarse como aquellos que tienen un impacto en la inocuidad, pero no directamente como los tiempos y temperaturas $(\mathrm{C}+)$.
La mayoría de los establecimientos cumplen parcialmente con los aspectos abordados en el acta de verificación (tabla 2), que coincide con los resultados informados por Zavala (2015). Al evaluar las categorías de calificación ( , C, C +), de acuerdo con la sección acta, no se detectaron diferencias significativas $(\mathrm{p} \geq 0.05), \quad$ sin embargo, al realizarlo de acuerdo con las categorías SCIAN, las calificaciones $\mathrm{C}$ (aspectos intermedios) y $\mathrm{C}+$ (Aspectos específicos), presentan diferencias significativas $(p \leq 0.05)$, que reflejan que estadísticamente los puntos referidos al control específico para minimizar el riesgo de transmisión de enfermedades al consumidor, son los que menos se cumplen en promedio (66.17) ( tabla 1), en todos los establecimientos evaluados.

El promedio general de $\mathrm{R}$ fue de 68.86 puntos, de C fue de 72.91 y de $C+$ fue de 66.16. Mediante el análisis estadístico se revela que, $\mathrm{R}$ y $C$ presentan diferencias significativas ( $p$ $<0.05$ ); mientras que las diferencias entre $\mathrm{C}$ y $\mathrm{C}$ + son altamente significativas ( $p<0.01)$, mientras que los puntos de aspectos básicos de inocuidad ( $\mathrm{R}$ ) y los puntos de aspectos intermedios $(\mathrm{C}+)$ son estadísticamente similares.

Los siguientes establecimientos se destacan por categoría: en la sección $\mathrm{R}$, la calificación más alta 72.22 fue para cafeterías y similares; en el aspecto $\mathrm{C}$ el puntaje más alto correspondió con 77.24 a alimentos y bebidas alcohólicas y no alcohólicas y en $\mathrm{C}+75.58$ que corresponde a bares, cantinas y similares (tabla 1). Teniendo en cuenta que los procedimientos de saneamiento son un pilar indispensable en los servicios de alimentos, preocupa el hecho de que el $98.76 \%$ de los restaurantes cumple parcialmente con los aspectos de mantenimiento y limpieza, ya que esto no se realiza de acuerdo con el tipo de proceso y producto específico, que aumenta la probabilidad de contaminación cruzada y, por lo tanto, no garantiza las condiciones de seguridad en el medio ambiente alrededor de los alimentos. La capacitación respaldada por documentación obtuvo el puntaje más alto en el $51.85 \%$ de los establecimientos. 


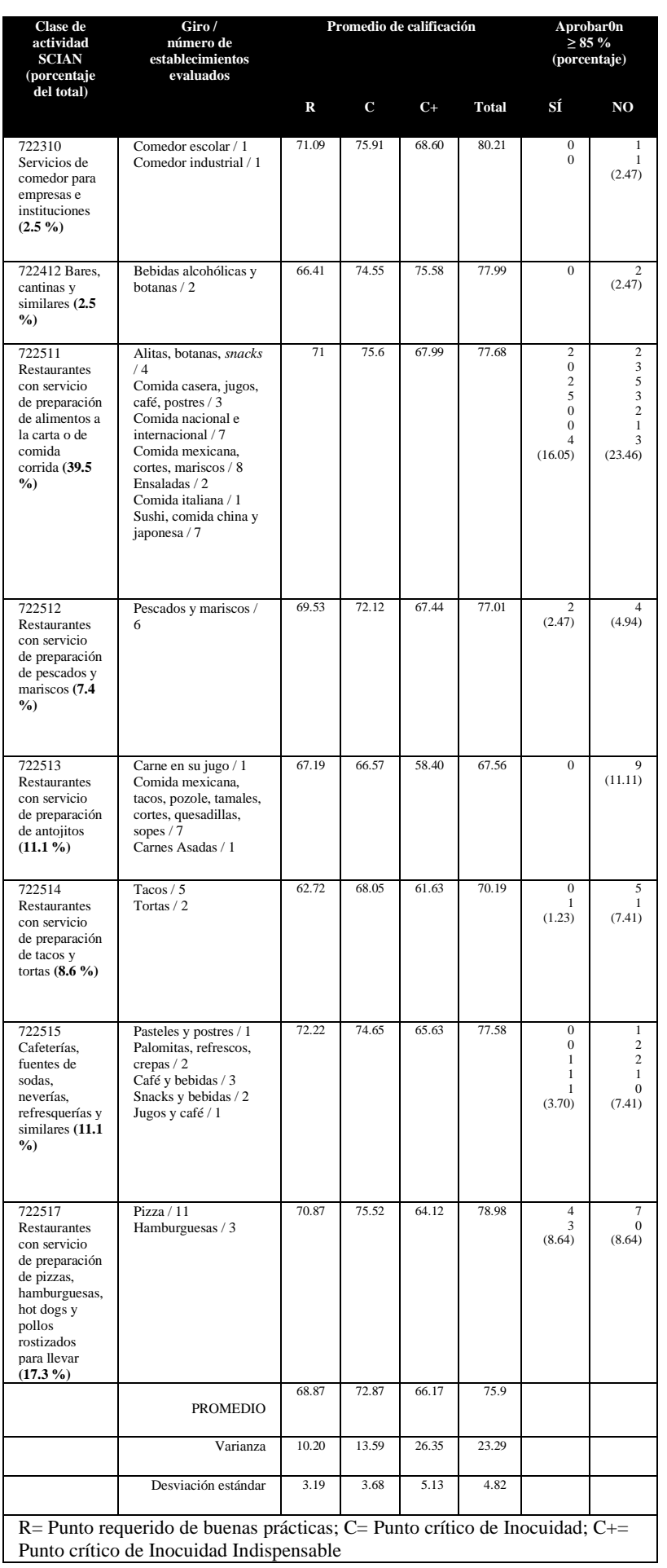

Tabla 1 Resultados de 81 establecimientos evaluados por medio del acta de verificación de la NOM-251-SSA12009 y la guía de autoevaluación del COFEPRIS

\begin{tabular}{|c|c|c|c|c|c|}
\hline & Cumplimiento & Total & Parcial & Nulo & $\begin{array}{c}\text { Puntaje } \\
\text { promedio }\end{array}$ \\
\hline \multirow{9}{*}{ 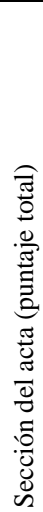 } & $\begin{array}{l}\text { Instalaciones y } \\
\text { áreas (12) }\end{array}$ & 9 & 72 & 0 & 8.3 \\
\hline & $\begin{array}{l}\text { Equipo y } \\
\text { utensilios (14) }\end{array}$ & 12 & 69 & 0 & 12.3 \\
\hline & Servicios (32) & 2 & 7 & 0 & $23.2 *$ \\
\hline & $\begin{array}{l}\text { Almacenamiento } \\
\text { (18) }\end{array}$ & 16 & 65 & 0 & 14 \\
\hline & $\begin{array}{l}\text { Control de } \\
\text { operaciones (48) }\end{array}$ & 0 & 81 & 0 & $28.9 *$ \\
\hline & $\begin{array}{l}\text { Materias primas } \\
\text { (10) }\end{array}$ & 35 & $\overline{46}$ & 0 & 8.4 \\
\hline & Envases (8) & 30 & 50 & 1 & 6 \\
\hline & $\begin{array}{l}\text { Agua en } \\
\text { contacto (6) }\end{array}$ & 18 & 63 & 0 & 4.2 \\
\hline & $\begin{array}{l}\text { Mantenimiento y } \\
\text { limpieza (54) }\end{array}$ & 1 & 80 & 0 & $37.4 *$ \\
\hline
\end{tabular}

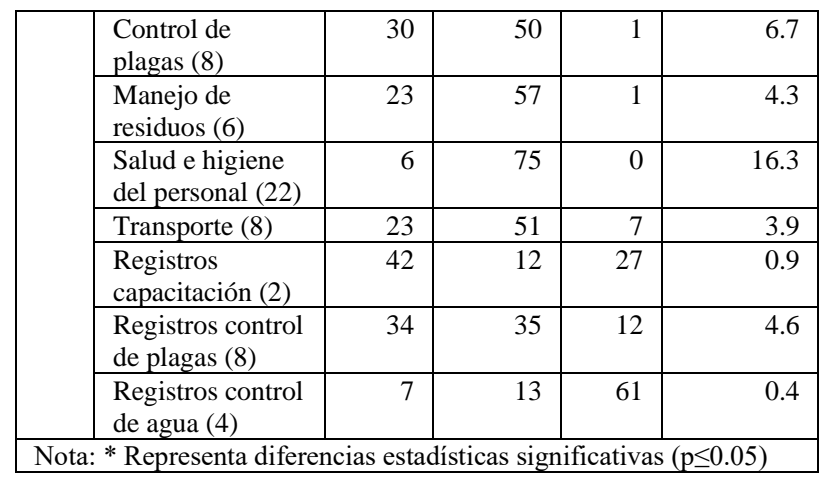

Tabla 2 Número de establecimientos que cumplieron por apartado de la Norma

\section{Agradecimiento}

El estudio fue financiado por la Universidad de Guadalajara.

\section{Conclusiones}

En términos generales, las condiciones y medidas aplicadas en la preparación y servicio de los alimentos no son suficientes para asegurar la calidad e inocuidad de los mismos, aun cuando se superaron las expectativas del $25 \%$ de aprobación. No importa la categoría en la que se clasifique el restaurante, importan los PPR implementados. Los restaurantes independientes (operaciones de una sola unidad) tienen mayores desviaciones críticas que los restaurantes de franquicia. Hay una suma de factores de riesgo en los procesos que podrían conducir a la aparición de riesgos bacteriológicos para la salud de los comensales. La mayoría de los servicios de alimentos evaluados no tienen un sistema de aseguramiento de la inocuidad correcto que permita cumplir con la NOM-251-SSA1-2009.

Existe una falta de conocimiento por parte de los responsables de los servicios de alimentos sobre los aspectos que deben cumplir de acuerdo con las reglamentaciones (el personal no está certificado), por lo que se requiere capacitación teórico-práctica especialmente para este propósito. Conocer la situación real en un contexto específico ayuda a la elaboración de estrategias más eficientes para asegurar la inocuidad alimentaria en los restaurantes.

Se requiere una investigación complementaria para identificar la causa raíz de todas las prácticas inapropiadas para la inocuidad alimentaria a fin de establecer las estrategias de intervención más efectivas que mejoren el sistema de protección de alimentos.

CAMPOS-BRAVO, Carlos Alberto, JIMÉNEZ-ORTEGA, Luis Alfonso, GONZÁLEZ-AGUILAR, Delia Guillermina y BARBARO, Bárbara. Diagnóstico higiénico-sanitario e identificación de factores de riesgo en restaurantes. Revista de Ciencias de la Salud. 2019 
La siguiente etapa de la investigación será el diseño de un curso de capacitación práctica (que se enfoque en: control de operaciones; control de calidad del agua; mantenimiento y limpieza/desinfección/; control de plagas; propuestas para posibles modificaciones operativas/estructurales), con estudios de caso recuperados de las visitas realizadas, buscando la colaboración de las autoridades sanitarias locales para desarrollar el material e implementar el curso en los mismos restaurantes que en el presente estudio obtuvieron los puntajes por debajo del $85 \%$. Lo anterior como parte del compromiso social que las instituciones educativas tienen con el entorno productivo.

\section{Referencias}

Angelo, K.M., Nisler, A.L., Hall, A.J., Brown, L.G. y Gould, L.H. (2017). Epidemiology of Restaurant-associated Foodborne Disease Outbreaks, United States, 1998-2013. Epidemiology \& Infection, 145 (3), 523-534. doi: $10.1017 / \mathrm{S} 0950268816002314$

Angulo, F.J., Jones, T.F. y Angulo, F.J. (2006). Eating in Restaurants: A Risk Factor for Foodborne Disease? Clinical Infectious Diseases, 43 (10), 1324-1328. doi: 10.1086/50 8540

Comisión Federal para la Protección contra Riesgos Sanitarios [COFEPRIS]. (2017). Marco normativo para alimentos. Guía de autoevaluación. Recuperado de https://www. gob.mx/cofepris/acciones-y-programas/marconormativo-para-alimentos Publicación: 25.10.2016. Ultima actualización: 14.02.2017.

Cormier, R.J., Mallet, M., Chiasson, S., Magnússon, H. y Valdimarsson, G. (2007). Effectiveness and Performance of HACCPbased Programs. Food Control, 18 (6), 665-671. doi: 10.1016/j.foodcont.2006.02.019

DiPietro, S., Haritchabalet, K., Cantoni, G., Iglesias, L., Mancini, S., Temperoni, A., Labanchi, J.L., Barbarossa, N., Garcia, M.T., Cofre, M., Rosales, S., Herrero, E., Bigatti, R., Orellana, O. y Larrieu, E. (2004). Vigilancia epidemiológica de enfermedades transmitidas por alimentos en la provincia de Rio Negro, Argentina, 1993-2001. Medicina (Buenos Aires), 64 (2), 120-124.
Djekic, I., Smigic, N., Kalogianni, E.P., Rocha, A., Zamioudi, L. and Pacheco, R. (2014). Food hygiene practices in different food establishments. Food Control, 39, 34-40. doi: 10.1016/j.foodcont.2013.10.035

Flórez, A.C., Rincón, C., Garzón, P., Vargas, N. y Enríquez, C. (2008). Factores relacionados con enfermedades transmitidas por alimentos en restaurantes de cinco ciudades de Colombia, 2007. Infectio, 12 (4), 255-266.

Food and Drug Administration [FDA]. (2018). FDA Report on the Occurrence of Foodborne Illness Risk Factors in Fast Food and Fullservice Restaurants, 2013-2014. Recuperado de https://www.fda.gov/media/117509/download

Garayoa, R., Yánez, N., Díez-Leturia, M., BesRastrollo, M. and Vitas, A.I. (2016). Evaluation of Prerequisite Programs Implementation and Hygiene Practices at Social Food Services through Audits and Microbiological Surveillance. Journal of Food Science, 81 (4), M921-M927. doi: 10.1111/ 1750-3841.13258

Harris, K.J., DiPietro, R.B., Murphy, K.S. y Rivera, G. (2014). Critical food safety violations in Florida: Relationship to location and chain vs. non-chain restaurants. International Journal of Hospitality Management, 38, 57-64. doi: 10.1016/j.ijhm. 2013.12.005

Harris, K.J., Murphy, K.S., DiPietro, R.B. y Rivera, G.L. (2015). Food safety inspections results: A comparison of ethnic-operated restaurants to non-ethnic-operated restaurants. International Journal of Hospitality Management, 46, 190-199. doi:10.1016/j.ijhm. 2015.02.004

Instituto Nacional de Estadística y Geografía [INEGI]. (2014). La industria restaurantera en México, Censos Económicos. Recuperado de http://www.canirac.org.mx/images/notas/files/ Mono_Restaurantera(1).pdf

Instituto Nacional de Estadística y Geografía [INEGI]. (2018). Sistema de Clasificación Industrial de América del Norte, México SCIAN, Recuperado de https://www.inegi.org. mx/app/scian/ 
Kopper, G., Calderón, G., Shneider, S., Domínguez, W. y Gutiérrez, G. (2009). Enfermedades transmitidas por alimentos y su impacto socioeconómico. Informe Técnico sobre Ingeniería Agrícola y Alimentaria 6. Estudios de caso en Costa Rica, El Salvador, Guatemala, Honduras y Nicaragua. FAO, Roma. Recuperado de http://www.fao.org/3/a-i0480s .pdf

Lachat, C., Nago, E., Verstraeten, R., Roberfroid, D., Van Camp, J. y Kolsteren, P. (2012). Eating out of home and its association with dietary intake: a systematic review of the evidence. Obesity Reviews, 13 (4), 329-346. doi: 10.1111/j.1467-789X.2011.00953.x

Leinwand, S.E., Glanz, K., Keenan, B.T. y Branas, C. C. (2017). Inspection Frequency, Sociodemographic Factors, and Food Safety Violations in Chain and Nonchain Restaurants, Philadelphia, Pennsylvania, 2013-2014. Public Health Reports, 132 (2), 180-187. doi: $10.1177 / 0033354916687741$

Luning, P.A., Marcelis, W.J., Rovira, J., Van der Spiegel, M., Uyttendaele, M. y Jacxsens, L. (2009). Systematic Assessment of Core Assurance Activities in a Company-specific Food Safety Management System. Trends in Food Science \& Technology, 20 (6-7), 300-312. doi: 10.1016/j.tifs.2009.03.003

Muguruza, C.N.E. (2019). Evaluación microbiológica de alimentos en una feria gastronómica,Lima - 2014. (Tesis de Maestría), Escuela de Posgrado, Universidad Nacional José Faustino Sánchez Carrión.

Nsoesie, E.O. Kluberg, S.A. y Brownstein, J.S. (2014). Online Reports of Foodborne Illness Capture Foods Implicated in Official Foodborne Outbreak Reports. Preventive Medicine, 67, 264-269. doi: 10.1016/j.ypmed.2014.08.003

Olea, A., Díaz, J., Fuentes, R., Vaquero, A. y García, M. (2012). Vigilancia de brotes de enfermedades transmitidas por alimentos en Chile. Revista Chilena de Infectología, 29 (5), 504-510. dx.doi:10.4067/S0716-10182012000 600004
Pérez, V.A.I., Lizárraga, C.J.N. y Medina, A.G. (2018). El inspector sanitario. Recomendaciones en restaurantes y bares en temporada vacacional. Revista COFEPRIS Protección y Salud, Recuperado de http://revistacofepris. salud.gob.mx/inter/2016/2/inspector. html

Pilco, H.J.G. (2019). Implementación de plan de higiene y saneamiento para la sección de salad bar. (Tesis de Ingeniería), Universidad Nacional Agraria la Molina, Facultad de Industrias Alimentarias.

Powell, L.M., Nguyen, B.T. y Han, E. (2012). Energy Intake from Restaurants: Demographics and Socioeconomics, 2003-2008. American Journal of Preventive Medicine, 43 (5), 498-504. doi: 10.1016/j.amepre.2012.07.041

Reske, K.A., Jenkins, T., Fernandez, C., Van Amber, D. y Hedberg, C.W. (2007). Beneficial Effects of Implementing an Announced Restaurant Inspection Program. Journal of Environmental Health, 69 (9), 27-34.

Rojas, H.L.M. (2019). Diseño e implementación de las bases de un modelo de sistema integrado de gestión para una microcerveceria artesanal. (Tesis de Maestría), Facultad de Ingeniería, Universidad Pontificia Javeriana, Cali.

Rodrigues, K.L., Silva, J.A. y Aleixo, J.A.G. (2012). Effect of the implementation of the Hazard Analysis Critical Control Point (HACCP) prerequisite program in an institutional foodservice unit in Southern Brazil. Ciência e Tecnologia de Alimentos, 32 (1), 196200. dx.doi: 10.1590/S0101-20612012 005000018

Secretaría de Salud [SS]. (2010). Norma Oficial Mexicana NOM-251-SSA1-2009, Prácticas de higiene para el proceso de alimentos, bebidas o suplementos alimenticios. Diario Oficial de la Federación. 01 Marzo 2010. Recuperado de http://www.dof.gob.mx/normasOficiales/3980/ salud/salud.htm

Statgraphics Centurion XVI version 16.2.04. 1992-2013. StatPoint Technologies, Inc.

Vera, J. y Trujillo, A. (2017). Escala mexicana de calidad en el servicio en restaurantes (EMCASER). Innovar Journal, 27 (63), 43-59. doi: 10.15446/innovar.v26n63.60665 
World Health Organization [WHO]. (2016). 10 facts on food safety. Recuperado de http://ww w.who.int/features/factfiles/food_safety/en/

World Health Organization [WHO]. (2019). Food safety. Recuperado de http://www.who. int/mediacentre/factsheets/fs399/en/

Zavala, N.M. (2015). Inocuidad alimentaria en restaurantes de hoteles Acapulco, Guerrero, México: franja de playa de la zona dorada. Revista Mexicana de Ciencias Agrícolas, 1, 517522. 